Screening bei erhöhtem Risiko

\title{
Bei Hausarztpatienten mehr auf Eisenmangel achten
}

\section{Unspezifische Symptome wie Müdigkeit, Schwäche, Haarausfall oder Kopf- schmerzen führen die Betroffenen meist zum Hausarzt. Hier könnte es sich loh- nen, das Labor zu bemühen. Erste Orien- tierung in Richtung Eisenmangelanä- mie bieten Blutbild und Serumferritin.}

_ Schätzungen der WHO zufolge leiden 19,8-33,6\% der europäischen Frauen vor der Menopause an einer Eisenmangelanämie. Um einen genaueren Überblick über die Häufigkeit zu erhalten, haben italienische Wissenschaftler Daten von Italienern, Spaniern, Belgiern und Deutschen untersucht. Grundlage der Erhebung waren längerfristige Aufzeichnungen nationaler Patientendatenbanken der Primärversorgung (z. B. IMS Health, in die in Deutschland 4.690 Allgemeinmediziner Informationen zu etwa 14 Mio. Patienten einspeisen).

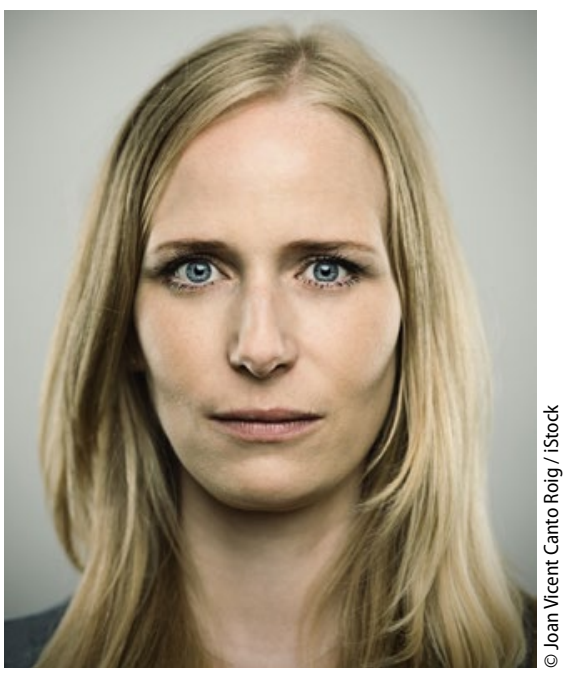

Blutbild und Ferritin bestimmen?

Die höchsten Inzidenzen zur Eisenmangelanämie zeichneten sich mit 12,42 bzw. 14,14/1.000 Personenjahre (PJ) im
Jahr 2011 in Deutschland und Spanien ab. In Deutschland war die Zahl der Neuerkrankungen zwischen 2007 und 2011 bei den Frauen auf 17,27 und bei den Männern auf 6,7/1.000 PJ angestiegen.

Abgesehen von dem zwei- bis vierfachen Anämierisiko der weiblichen Bevölkerung waren am häufigsten jüngere sowie alte Patienten betroffen. Auch im Zusammenhang mit gastrointestinalen Erkrankungen, Schwangerschaft, besonders starken Menstruationsblutungen sowie Einnahme von Acetylsalicylsäure oder Säureblockern traten vermehrt Eisenmangelanämien auf. Als häufigste Begleitsymptome zeigten sich allgemeine Schwäche, Kopfschmerzen, Xerostomie, Reizbarkeit und Alopezie.

Die Eisenmangelanämie wurde in früheren Erhebungen möglicherweise unterschätzt, so die Autoren. Sie empfehlen, dem Thema in der Hausarztpraxis mehr Aufmerksamkeit zu schenken und Patienten mit erhöhten Risiken zu screenen bzw. deren Therapieerfolge sorgfältig zu überwachen. $\quad$ - St

- Levi M et al. European Journal of Haematology 2016 online 7. Mai, doi: 10.1111/ejh.12776

\section{Hoher Blutverlust auf der Aschenbahn}

Mehr als jede vierte Frau in der europäischen Allgemeinbevölkerung leidet unter sehr starken Menstruationsblutungen. Auch bei Sportlerinnen ist dieses Problem weit verbreitet. Einer britischen Studie zufolge findet dies unter den Frauen selbst allerdings oft nur geringe Beachtung. Dabei reichen die Folgen von Eisenmangelanämien bis zur verringerten Leistungsfähigkeit, die unter Trainings- und Wettkampfbedingungen besonders zu Buche schlägt. In der Untersuchung wurden 789 sportliche Frauen ( $\geq 90$ Minuten regelmäßiges Training/ Woche) in sozialen Medien online zu Trainingspensum, Gesundheitszustand, Supplementationstherapien sowie Beeinträchtigungen durch die Menstruation befragt. Die gleichen Fragen beantworteten 1.073 Teilnehmerinnen des LondonMarathon 2015 in persönlichen Interviews. 54\% der online befragten Frauen und $36 \%$ der Marathonläuferinnen berichteten über Menorrhagien. Über die Hälfte der Sportlerinnen fühlte sich dadurch bei ihren körperlichen Aktivitäten zwar beeinträchtigt, aber die wenigsten hatten deshalb einen Arzt aufgesucht. Insgesamt 32\% gaben eine Anämie in der Vorgeschichte zu Protokoll, jede Zweite hatte bereits Eisen supplementiert.

\section{Anämie verändert Herzfunktion in der Schwangerschaft}

Eine Eisenmangelanämie in der Schwangerschaft kann sich in verschiedenen Veränderungen der Herzfunktion widerspiegeln. Bei $90 \%$ der anämischen Frauen im zweiten Schwangerschaftstrimenon traten in einer indischen Studie Tachykardien und Abnormitäten im EKG auf. So verringerte sich bei Anämie (Hämoblobin 7-9,9 g/dl, Serumferritin $<4,6 \mathrm{ng} / \mathrm{ml}$ ) die QRS-Dauer signifikant und das QTc-Intervall nahm gegenüber nicht anämischen Schwangeren zu. Auch abgeflachte und negative T-Wellen kamen häufiger vor.

- Tangeda PR et al. J Clin Diagn Res. 2016;10:CC16-8

\section{Anämie-Inzidenz unter Chemotherapie}

Die Mehrzahl der Krebspatienten wird auch heute noch unter der Behandlung anämisch. Bei 89,5\% von insgesamt 4.426 Patienten mit soliden Tumoren sank das Hämoglobin, einer US-Studie zufolge, während der Chemotherapie unter den Normwert. 26\% der Patienten mit Kolorektalkarzinom und 59\% der Frauen mit Ovarialkarzinom entwickelten eine Anämie mit einem $\mathrm{Hb}<10 \mathrm{~g} / \mathrm{dl}$. Zudem variierte die Inzidenz einer 2+-gradigen Anämie je nach Behandlungsart. Sie reichte von $18 \%$ bei Brustkrebspatientinnen unter Cyclophosphamid plus Docetaxel bis $60 \%$ bei Frauen mit Ovarialkarzinom, die Carboplatin und Paclitaxel erhielten. 